\title{
Study of Higher Harmonics of Cosmic Ray Intensity on Quiet Days at Deep River Neutron Monitoring Station
}

\author{
M. K. Richharia \\ Department of Physics, Govt. Science College (Autonomous) Jabalpur (M.P.), India
}

\section{Email address:}

mkrichharia@yahoo.com

\section{To cite this article:}

M. K. Richharia. Study of Higher Harmonics of Cosmic Ray Intensity on Quiet Days at Deep River Neutron Monitoring Station. International Journal of Astrophysics and Space Science. Special Issue: Modulation of Cosmic Rays, Inter Planetary Magnetic Field, Magnetic Storm, Large and Small Amplitude Wave Train. Vol. 3, No. 2-1, 2015, pp. 4-6. doi: 10.11648/j.ijass.s.2015030201.12

\begin{abstract}
The cosmic ray (CR) intensity data recorded with Deep River Neutron Monitoring Station have been investigated on quietest days (QD) for higher harmonics of daily variation during solar cycle 21 and 22. It has been observed that inspite of the abrupt change in the amplitude and phase of tri-diurnal and quart diurnal anisotropy in CR intensity, the amplitude of third harmonic is quite significant throughout the period of investigation with larger amplitude during the year 1980 and 1985 . Thus, tri-diurnal anisotropy clearly shows 11 year variation at mid latitude neutron monitoring station.
\end{abstract}

Keywords: Cosmic Rays/ Higher Harmonics/Geo-magnetically Quiet Days

\section{Introduction}

The spatial anisotropy of the galactic cosmic ray intensity in the interplanetary space manifests itself as daily variation with a period of 24 hours (and its higher harmonics) due to the rotation of the Earth in the course of a day. The Power Spectrum analysis as well as the Fourier analysis of the long term data of the 24-hour values of cosmic ray (CR) intensity observed by Earth based detectors have provided confirmatory existence along with the characteristics of the first three harmonics of daily variation of extra terrestrial origin $[1,2]$. However, the amplitude of the fourth harmonics is still controversial [3,7], Moreover, it has been observed that the amplitude and phase of tri-diurnal variation of $\mathrm{CR}$ intensity on quiet days also vary considerably from one period to another. On the long term behaviour of the first three harmonics showed that high degree of year to year variability exists, a trend with solar activity was evident.

The higher harmonic components of daily variation, which represents may be anisotropy produced by cosmic ray streaming flows due to local particle gradients. This is very important study for understanding the electromagnetic environment of the interplanetary region. Thus, the studies of the higher harmonics in the daily variation of cosmic rays provide valuable information as to the nature of the cosmic ray modulation in the heliosphere.

\section{Analysis of the Data}

The pressure corrected hourly CR intensity data (corrected for meteorological effects) on geo-magnetically five quietest days (QD) in every month for Deep River (Lat:46.06N; cutoff rigidity: $1.02 \mathrm{GV}$; Longitude $282.5^{\circ} \mathrm{E}$; Altitude $145 \mathrm{~m}$ ) neutron monitoring station and for the period 1978-94 have been used in Fourier analysis. After applying the trend correction, such a set of data have been subjected to Harmonic analysis for each day [8]. The average values of the amplitude and phase (local time of the station) of the third (Tri-diurnal) and fourth harmonics (Quart diurnal) on yearly basis have been obtained. According to solar geophysical data five quietest days are selected in a month; thus 60 quietest days are obtained in a year. These days are called international quiet days (QD). The days with extra ordinary large amplitude if any, have not been considered. Further, the variation in the tri-diurnal anisotropy with the reversal of polarity of solar magnetic field (PSMF) on 60QD has also been investigated. Also all those days are discarded having more than three continuous hourly data missing.

\section{Results and Discussion}

The yearly average amplitude and phase of the third harmonics of daily variation for Deep River Neutron Monitoring Station have been plotted in Fig. 1 and 2 during 
the period 1978-94 on quiet days. It is quite apparent from Fig. 1 that the amplitude of third harmonics of daily variation has quite abruptly increased during the years 1980 and 1985. The likely cause for such type of variation could be the changing of geomagnetic threshold cut off rigidly from $1.02 \mathrm{GV}$ to 1.15 and 1.12 in 1980 and 1985 respectively [9.11] as it has been discussed in the case of change of diurnal anisotropy of cosmic ray intensity on QD [12]. These type of variation in the amplitude' of the tridiurnal anisotropy on QD may be attributed to the change in the rigidity spectrum.

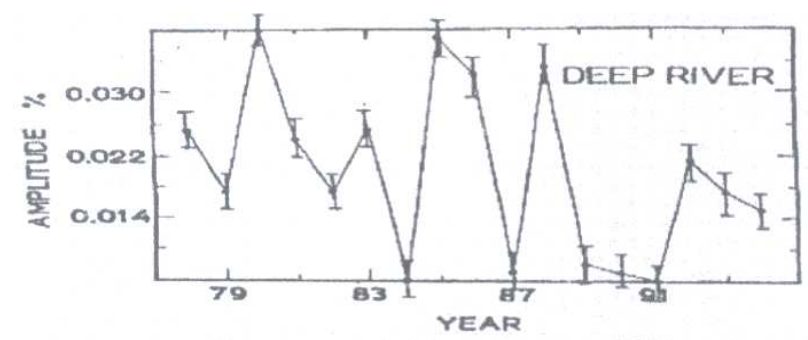

Fig. 1. The annual amplitude (\%) of tri-diurnal anisotropy of cosmic ray intensity on quiet days during 1978-1994 at Deep River Neutron Monitoring Station.

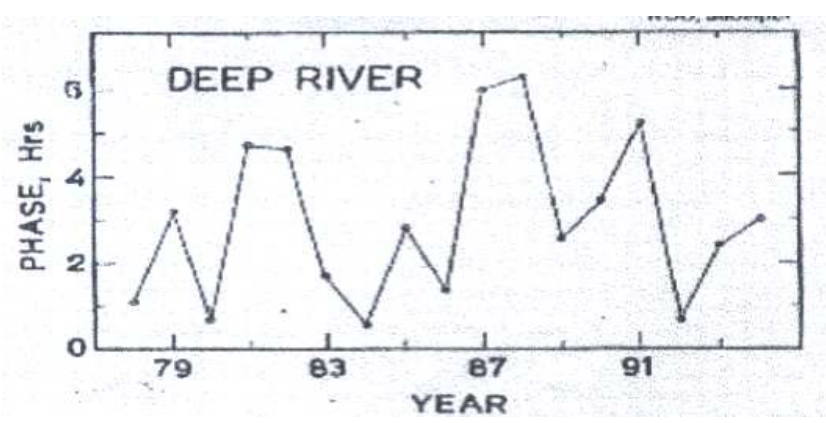

Fig. 2. The annual phase (hrs) of tri-diurnal anisotropy of cosmic ray intensity on quiet days during 1978-1994 at Deep River Neutron Monitoring Station.

The amplitude of tri-diurnal anisotropy on QD has shown an exceptionally small value during 1987 , which is a period of minimum solar activity [13]. The amplitude of third harmonics of daily variation on QD is observed to be significantly low during 1981 as well as in 1990, which coincides with phase reversal of the solar poloidal magnetic field [14].

It is observed from Fig.2 that there is no systematic change in the phase of third harmonics of daily variation of cosmic ray intensity on quiet days. However, a slight change in the value of tri-diurnal phase is observed, when the solar polar magnetic field reversed its polarity during the periods 1979-80 and 1990-91 [14]. It shows that the phase of tridiurnal anisotropy on quiet days has nearly the same value at both sides of reversal period. But in both the cases during the succeeding years, i.e., 1980-81 and 1991-92, the change in the phase of tri-diurnal anisotropy of CR intensity has been found quite significant [15]. This supports 11 year type variation in tri-diurnal anisotropy of $\mathrm{CR}$ intensity on quiet days [16-18].
It is quite apparent from the Fig.3 that there is no systematic change in the amplitude in the fourth harmonics of daily variation on quiet days, the amplitude during the year 1979 and 1985 has quite abruptly increased. Further the year 1987, the amplitude has small value, which is a period of minimum solar activity. The amplitude during the years 1980 and 1991 has been observed same value, which is period of high solar activity in the solar cycle 21 and 22 . This also confirm the 11 year type variation occur in the fourth harmonics of daily variation.

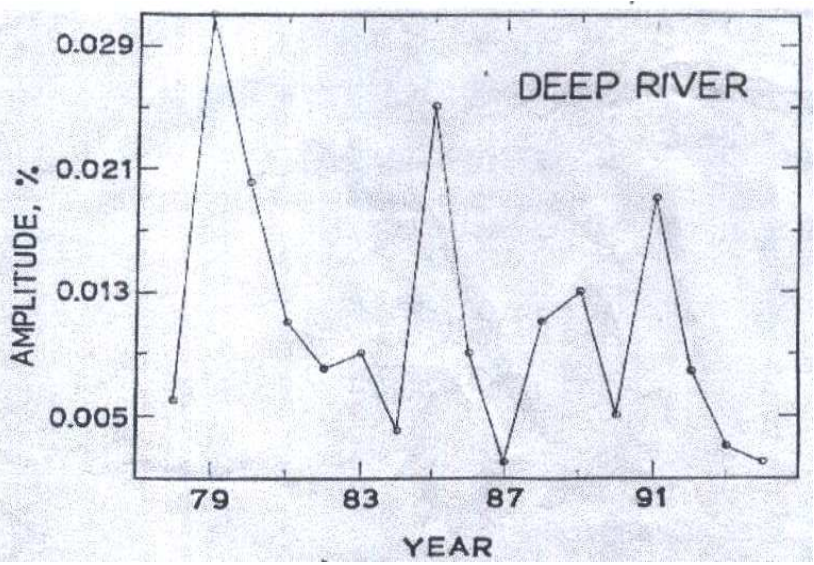

Fig. 3. The annual amplitude (\%) of quart-diurnal anisotropy of $C R$ intensity on quiet days during 1978 - 1994 at Deep River Neutron Monitoring Station.

It is quite apparent from fig 4, that there is no systematic change in the phase of fourth harmonics, the phase in the year 1981 and 1992 has occurred in the same direction, which indicate likely 11 year type variation [15].

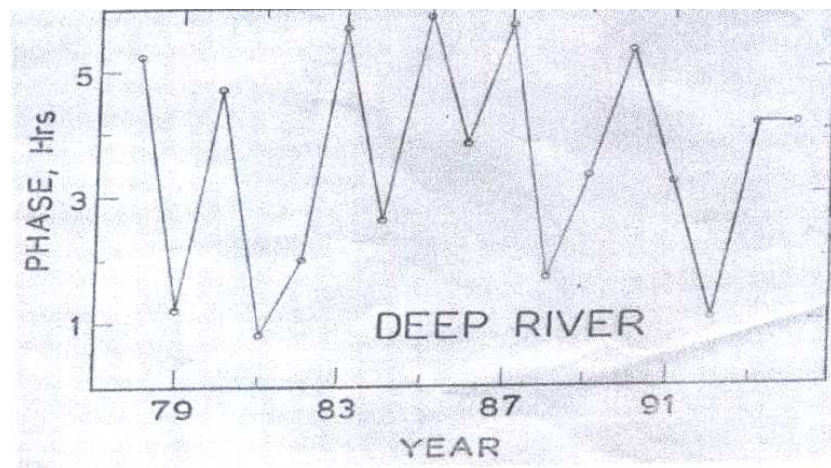

Fig. 4. The annual phase (hrs) of quart-diurnal anisotropy of CR intensity on quiet days during 1978 - 1994 at Deep River Neutron Monitoring Station.

\section{Conclusions}

Following conclusion may be drawn from the present investigation.

1. The amplitude of tri-diurnal anisotropy of CR intensity on QD has abruptly increased due to changing of threshold cutoff rigidity of the station.

2. The amplitude of tri-diurnal and quart diurnal anisotropy on QD having small value during the year 
1987. i.e., which is the of minimum solar activity period.

3. The amplitude of third harmonics of daily variation on QD during 1981 and 1990 is significantly low, which coincides with the phase reversal of solar poloidal field.

4. The tri-diurnal and quart diurnal anisotropy of $\mathrm{CR}$ intensity on QD has shown long term variation .i.e., 11 year variations.

\section{Acknowledgements}

The authors are indebted to various experimental groups; in particular, Profs. M. Bercovitch, K.Nagashima and Miss Aoi Inoue for providing neutron monitor data.

\section{References}

[1] Fujii,A., Nagashima, K., Fujimoto, K., Ueno, H. and Kondo, ICRC, Hobart, Tasmania, 2, 666 (1971).

[2] Ahluwalia, H.S. and Singh, S. Proc. 13th Int. Cosmic Ray Conf, Australia, 2: 948 (1973 b).

[3] Pomerantz, M.A. and Duggal, S.P. Space Sci. Rev., 12: 75 (1971).

[4] Rao, U.R. Space Sci. Rev., 12 : 719 (1972).

[5] Venkatesan, D. and Badruddin. Space Sci. Rev., 52: 121 (1990).

[6] Ahluwalia, H.S. and Singh, S. Proc. 13th Int. Cosmic Ray Conf., Australia, 5: 3129 (1973 a).
[7] Agrawal, S.P. J. Geophys. Res., 86: 10115 (1981).

[8] Yadav, R.S. and Naqvi, T. H. Tech. Note No. 1, A.M.U. Aligarh (1973).

[9] Smart, D.F. and Shea, M.A. 20th Int. Cosmic Ray Conf., Moscow 4: 204 (1987).

[10] Shea, M.A. and Smart, D.F. 18th Int. Cosmic Ray Conf., Bangalore, 3:411. (1983).

[11] Shea, M.A. and Smart, D.F. 27th hit. Cosmic Ray Conf., Hemberg, 3:4063 (2001).

[12] Kumar, S., Gulati, U., Khare, D. and Richharia, M.K. Bull Astronomical Soc. India, $21: 395$ (1993).

[13] Kumar, S., Richharia, M.K., Chauhan, M.L., Gulati, U., Khare, D.K. and Shrivastava, S.K. 24th Int. Cosmic Ray Conf., Roma Italy, 4 : 623 (1995).

[14] Kumar, S., Shrivastava, S.K., Dubey, S.K., Richharia, M.K. and Gulati, U. Ind. J. Radio and Space Phys., 27 : 236 (1998).

[15] El Bofie, Sabbah, M.A., Darwish, A.A, and Bishra, A.A. 24th Int. Cosmic Ray Conf., Roma, Italy, 4: 619 (1995).

[16] Richharia, M.K., Shrivastava, S.K. and Kumar, S. J. Pure and Applied Phys., 11, 1: 11(1999).

[17] Richharia, M.K., Kumar S. and Shrivastava S.K. Res. J. (Sci.) R.D. University, Jabalpur Vol. 7. No. 2.195 (2000).

[18] Richharia, M.K. 27th Int. Cosmic Ray conference, Hemberg, 3, 3744 (2001) 